\title{
Arthrite dans la population autochtone canadienne : différences entre le Nord et le Sud en ce qui a trait à la prévalence et aux corrélats
}

\author{
C. Ng, M. Sc. (1); S. Chatwood, M. Sc. (2,3); T.K. Young, M.D., Ph. D. (3)
}

* Cet article fait l'objet d'une publication conjointe par Preventing Chronic Disease et Maladies chroniques au Canada. Maladies chroniques au Canada en est le premier éditeur, Preventing Chronic Disease le second.

\section{Résumé}

Contexte : Les données sur l'arthrite et les autres troubles musculosquelettiques chez les Autochtones sont rares. Les données d'enquête montrent que l'arthrite et le rhumatisme figurent parmi les troubles chroniques les plus fréquemment signalés et que leur prévalence est plus élevée chez les Autochtones que chez les non-Autochtones.

Objectif : Décrire le fardeau de l'arthrite au sein de la population autochtone du nord du Canada et démontrer l'impact social et l'importance sur le plan de la santé publique de cette maladie.

Méthodologie : À partir de données transversales recueillies auprès de plus de 29000 personnes autochtones âgées de 15 ans et plus et ayant participé à l'Enquête auprès des peuples autochtones de 2006, les différences régionales relatives à la prévalence de l'arthrite ont été évaluées, de même que les liens avec l'utilisation des services de santé et certains facteurs de risque et de comorbidité.

Résultats : Dans les trois territoires du Nord (le "Nord »), la prévalence de l'arthrite était de $12,7 \%$, alors qu'elle était de $20,1 \%$ dans les provinces (le "Sud »). Dans le Nord comme dans le Sud, la prévalence était plus élevée chez les femmes que chez les hommes. Elle demeure moins élevée chez les Inuits qu'au sein des autres groupes autochtones. Les personnes atteintes d'arthrite étaient davantage susceptibles de fumer, d'être obèses, de souffrir d'autres maladies chroniques et d'être sans emploi. Les Autochtones atteints d'arthrite ont utilisé le système de santé plus souvent que ceux ne souffrant pas de cette maladie.

Conclusion : Les conclusions concernant l'arthrite et d'autres maladies chroniques chez les Autochtones ainsi que la reconnaissance des différences entre le Sud et le Nord seront profitables pour la planification de programmes et l'établissement de nouvelles priorités en matière de promotion de la santé.

Mots-clés : arthrite, Autochtones, Nord canadien, Inuits, Premières nations, Métis, Indiens d'Amérique du Nord, Enquête auprès des peuples autochtones

\section{Introduction}

Les données sur l'arthrite et les autres troubles musculosquelettiques chez les Autochtones sont peu nombreuses, et celles qui existent proviennent de secteurs géographiques limités, surtout de l'Alaska, de la Colombie-Britannique et du Manitoba $^{1-3}$. Plusieurs enquêtes nationales - l'Enquête régionale longitudinale sur la santé des Premières Nations (ERS) ${ }^{4,5}$, l'Enquête sur la santé dans les collectivités canadiennes (ESCC) ${ }^{6,7}$ et l'Enquête auprès des peuples autochtones (EPA) ${ }^{8,9}$
- ont permis de recueillir, auprès d'adultes autochtones, des données sur la prévalence de l'arthrite, du rhumatisme et d'autres troubles musculosquelettiques comme les maux de dos. En règle générale, ces enquêtes ont révélé que l'arthrite et le rhumatisme figurent parmi les troubles chroniques les plus fréquemment signalés, que leur prévalence est plus élevée chez les Autochtones que chez les non-Autochtones au Canada et que cette prévalence s'accroît. Par exemple, d'après les résultats de l'EPA, la prévalence brute serait passée de $15 \%$ en 1991 à $19 \%$ en $2001^{8,9}$, et d'après les résultats de l'ERS, la prévalence ajustée selon l'âge serait passée de $22 \%$ en 1997 à $25 \%$ en 2002-2003 ${ }^{4,5}$. (Remarque : les résultats de ces différentes enquêtes ne peuvent être comparées directement, parce qu'elles portent sur des groupes autochtones différents et que des populations-types différentes ont été utilisées pour l'ajustement des taux selon l'âge). L'arthrite est aussi un facteur dans plus de la moitié des incapacités déclarées par les membres des Premières nations au Canada ${ }^{5}$.

L'incapacité attribuable à l'arthrite peut être exacerbée dans le Nord canadien par de mauvaises conditions météorologiques, des infrastructures inadéquates et des moyens de transport pas toujours fiables. L'arthrite mine la capacité des Autochtones qui en sont atteints à participer aux activités traditionnelles, comme l'artisanat traditionnel et la cueillette d'aliments. L'isolement géographique de nombreuses communautés restreint l'accès aux services de spécialistes. Le contexte culturel est une dimension supplémentaire : des orientations propres aux diverses régions

\section{Rattachement}

1. Institute of Medical Science, Université de Toronto, Toronto (Ontario)

2. Institute for Circumpolar Health Research, Yellowknife (Territoires du Nord-Ouest)

3. Dalla Lana School of Public Health, Université de Toronto, Toronto (Ontario)

Correspondance : T. Kue Young,155, rue College, bureau 547, Toronto (Ontario) M5T 3M7; tél. : 416-978-6459; téléc. : 416-946-8055; courriel : kue.young@utoronto.ca 
et de vastes partenariats sont nécessaires à la planification et à la mise en œuvre de services de santé et de systèmes de soutien adaptés sur le plan culturel. Il faut notamment tenir compte (sans s'y limiter) de l'importance de l'accès aux remèdes et aux guérisseurs traditionnels, des langues parlées et de l'organisation des services de soutien dans les communautés.

Le présent article brosse le tableau du fardeau de l'arthrite chez les Autochtones au Yukon, dans les Territoires du NordOuest et au Nunavut, les trois territoires du Nord canadien. À partir de données publiées récemment dans le cadre de l'Enquête auprès des peuples autochtones de 2006 (EPA de 2006) ${ }^{10}$, les différences régionales - c'est-à-dire entre ces trois territoires (le " Nord ») et les dix provinces (le "Sud») - relatives à la prévalence de l'arthrite ont été évaluées, de même que les liens avec l'utilisation des services de santé et certains facteurs de risque et de comorbidité.

\section{Méthodologie}

Des données transversales ont été recueillies auprès de plus de 29000 personnes authochtones âgées de 15 ans et plus (Inuits, Métis ou membres des Premières nations vivant hors réserve) et ayant participé à l'EPA de 2006 (tableau 1). Statistique Canada a réalisé l'EPA en tant qu'enquête postcensitaire afin de recueillir des données sur les conditions économiques et sociales des Autochtones vivant au Canada.

Dans le cadre de l'EPA de 2006, on a demandé aux répondants si un médecin, une infirmière ou un autre professionnel de la santé leur avait déjà dit qu'ils souffraient d'arthrite ou de rhumatisme. Les corrélations entre l'arthrite et diverses variables démographiques, socioéconomiques, comportementales et liées aux soins de santé ont été examinées séparément pour les trois territoires et les dix provinces, non pas pour confirmer des hypothèses étiologiques, mais plutôt pour démontrer l'impact social du fardeau de l'arthrite et sa signification sur le plan de la santé publique. L'obésité, définie par un indice de masse corporelle (IMC)

TABLEAU 1

Nombre de répondants de $\mathbf{1 5}$ ans et plus par région et groupe autochtone dans le Nord ${ }^{\mathrm{a}}$ et le Sud ${ }^{\mathrm{b}}$ du Canada

\begin{tabular}{lcccr}
\hline & \multicolumn{2}{c}{ Nord $^{\mathrm{a}}$} & \multicolumn{2}{c}{ Sud $^{\mathrm{b}}$} \\
& Hommes & Femmes & Hommes & Femmes \\
\hline Premières nations & 380 & 420 & 5260 & 6680 \\
Inuits & 1630 & 1650 & 830 & 880 \\
Autres $^{c}$ & 270 & 260 & 4990 & 5870 \\
\hline
\end{tabular}

Remarque : Ces résultats sont non pondérés, et les nombres ont été arrondis à la dizaine la plus proche.

${ }^{a}$ Les trois territoires du Nord canadien : Nunavut, Territoires du Nord-Ouest et Yukon

b Les dix provinces canadiennes : Colombie-Britannique, Alberta, Saskatchewan, Manitoba, Ontario, Québec, Nouveau-Brunswick, Terre-Neuve-et-Labrador, Nouvelle-Écosse et Île-du-Prince-Édouard.

' Répondants d'ascendance Métis ou plus d'un groupe autochtone

de $30 \mathrm{~kg} / \mathrm{m}^{2}$ ou plus, et le tabagisme sont des facteurs de risque déjà connus pour l'arthrite ${ }^{11,12}$, et dans le cadre de l'EPA de 2006, on a demandé aux répondants de fournir leur taille et leur poids ainsi que leurs antécédents et leurs habitudes en matière de tabagisme. L'arthrite est également associée à une réduction de l'emploi et à des restrictions relatives au travail chez l'adulte ${ }^{13}$; dans le cadre de l'EPA, on a demandé aux répondants s'ils avaient effectué un travail rémunéré ou à leur compte au cours de la semaine précédente.

Nous avons établi la prévalence de l'arthrite pour les trois groupes en demandant aux répondants si leurs ancêtres appartenaient aux groupes suivants autochtones : Indiens de l'Amérique du Nord, Métis ou Inuits [les répondants pouvaient indiquer plus qu'un groupe]. Les personnes ayant choisi le groupe "Indiens de l'Amérique du Nord » composent le groupe des "Premières nations ", celles ayant choisi le groupe « Inuit » composent le groupe des Inuits et toutes les autres, notamment celles ayant choisi le groupe " Métis » et celles ayant répondu " faire partie de plus d'un groupe ", ont été regroupées en une catégorie "Autres », étant donné la petite taille des échantillons de chacun de ces groupes dans le Nord. Seules les proportions de prévalence brute ont été rapportées; la prévalence ajustée selon l'âge n'a pas été calculée, puisque l'ensemble de données ne renfermait pas de renseignements sur des personnes non autochtones qui auraient pu être utilisés à des fins de comparaison. En outre, la comparaison des résultats de cette étude avec les taux de prévalence ajustée selon l'âge déjà publiés aurait été difficile, compte tenu de l'utilisation de populations types différentes. Enfin, la prévalence brute permet de rendre compte du fardeau de la maladie avec exactitude en vue de planifier les programmes en santé publique.

Les analyses ont été effectuées à l'aide de la version 9.2 de SAS (SAS Institute, Cary, Caroline du Nord). Comme l'EPA de 2006 était fondée sur un plan d'enquête complexe, des poids d'enquête ont été utilisés pour toutes les analyses, et la variance a été estimée à l'aide de la méthode bootstrap, avec les 1000 poids bootstrap fournis par Statistique Canada. Toutes les proportions ont été établies conformément aux lignes directrices de Statistique Canada relativement à l'arrondissement. Les intervalles de confiance (IC) ont été calculés à partir de données non arrondies. Pour connaître la méthodologie détaillée, il faut s'adresser à Statistique Canada ${ }^{10}$. Les corrélations entre l'arthrite et diverses variables ont été établies au moyen d'analyses de régression logistique ajustées selon l'âge et le sexe.

\section{Résultats}

\section{Prévalence}

Pour les trois groupes autochtones combinés, la prévalence brute de l'arthrite ou du rhumatisme est de $12,7 \%$ (IC à $95 \%$ : 12,5 à 13,0) dans les territoires et de 20,1\% dans les provinces (IC à $95 \%$ : 19,9 à 20,3). La prévalence de l'arthrite est plus élevée chez les femmes que chez les hommes, et ce, dans le Nord comme dans le Sud. La prévalence chez les Inuits est inférieure à celle mesurée chez les membres des Premières nations et les autres groupes 
TABLEAU 2

Prévalence brute de l'arthrite et du rhumatisme chez les Autochtones de 15 ans et plus dans le Nord et le Sud du Canada

\begin{tabular}{|c|c|c|c|c|c|c|}
\hline & \multicolumn{3}{|c|}{ Nord $^{a}$} & \multicolumn{3}{|c|}{ Sudb } \\
\hline & $\begin{array}{l}\text { Prévalence (\%) } \\
\text { (IC à } 95 \%)\end{array}$ & $\begin{array}{l}\text { Prévalence (\%) } \\
\text { (IC à } 95 \%)\end{array}$ & $\begin{array}{l}\text { Prévalence (\%) } \\
\text { (IC à } 95 \% \text { ) }\end{array}$ & $\begin{array}{l}\text { Prévalence (\%) } \\
\text { (IC à } 95 \%)\end{array}$ & $\begin{array}{l}\text { Prévalence (\%) } \\
\text { (IC à } 95 \% \text { ) }\end{array}$ & $\begin{array}{l}\text { Prévalence (\%) } \\
\text { (IC à } 95 \%)\end{array}$ \\
\hline $\begin{array}{l}\text { Tous les groupes } \\
\text { autochtones }\end{array}$ & $10,2 \quad(9,8-10,6)$ & $15,2 \quad(14,7-15,6)$ & $12,7 \quad(12,5-13,0)$ & $16,2 \quad(16,0-16,5)$ & $23,3 \quad(23,0-23,5)$ & $20,1 \quad(19,9-20,3)$ \\
\hline Autres & $10,7 \quad(9,6-11,8)$ & $15,4 \quad(14,2-16,6)$ & $12,9 \quad(12,1-13,8)$ & $18,0 \quad(17,7-18,4)$ & $23,5 \quad(23,2-23,9)$ & $21,0 \quad(20,7-21,2)$ \\
\hline De 15 à 24 ans & $(1,5-2,1)$ & $(2,3-3,0)$ & $(2,0-2,4)$ & $(2,7-3,2)$ & $(4,2-4,8)$ & $(3,5-4,0)$ \\
\hline De 25 à 44 ans & $6,7 \quad(6,3-7,2)$ & $10,6 \quad(9,9-11,2)$ & $(8,3-9,1)$ & $10,5 \quad(10,2-10,8)$ & $15,0 \quad(14,7-15,4)$ & $13,1 \quad(12,9-13,3)$ \\
\hline De 45 à 64 ans & $20,9 \quad(19,7-22,0)$ & $29,5 \quad(28,3-30,6)$ & $25,3 \quad(24,5-26,1)$ & $29,0 \quad(28,4-29,6)$ & $40,0 \quad(39,5-40,6)$ & $35,0 \quad(34,6-35,4)$ \\
\hline
\end{tabular}

Abréviations : IC, intervalle de confiance

${ }^{a}$ Les trois territoires du Nord canadien : Nunavut, Territoires du Nord-Ouest et Yukon

${ }^{\text {b } L e s ~ d i x ~ p r o v i n c e s ~ c a n a d i e n n e s ~: ~ C o l o m b i e-B r i t a n n i q u e, ~ A l b e r t a, ~ S a s k a t c h e w a n, ~ M a n i t o b a, ~ O n t a r i o, ~ Q u e ́ b e c, ~ N o u v e a u-B r u n s w i c k, ~ T e r r e-N e u v e-e t-L a b r a d o r, ~ N o u v e l l e-E ́ c o s s e ~ e t ~}$ Île-du-Prince-Édouard.

TABLEAU 3

Prévalence brute des risques sanitaires associés à l'arthrite chez les Autochtones de 15 ans et plus dans le Nord ${ }^{\mathrm{a}}$ et le Sud ${ }^{\mathrm{b}}$ du Canada

\begin{tabular}{|c|c|c|c|c|}
\hline & \multicolumn{2}{|c|}{ Nord $^{a}$} & \multicolumn{2}{|c|}{ Sud ${ }^{b}$} \\
\hline & Personnes atteintes d'arthrite & Personnes non atteintes & Personnes atteintes d'arthrite & Personnes non atteintes \\
\hline & $\begin{array}{l}\text { Prévalence (\%) } \\
\text { (IC à } 95 \% \text { ) }\end{array}$ & $\begin{array}{l}\text { Prévalence (\%) } \\
\text { (IC à } 95 \% \text { ) }\end{array}$ & $\begin{array}{l}\text { Prévalence (\%) } \\
\text { (IC à } 95 \% \text { ) }\end{array}$ & $\begin{array}{l}\text { Prévalence (\%) } \\
\text { (IC à } 95 \% \text { ) }\end{array}$ \\
\hline Tabagisme quotidien & $44,8(43,6-46,1)$ & $51,6(51,1-52,1)$ & $36,2(35,8-36,7)$ & $29,6(29,4-29,8)$ \\
\hline Obésité (IMC $\geq 30$ ) & $36,7(35,4-38,0)$ & $23,7(23,2-24,2)$ & $33,4(32,9-33,8)$ & $22,6(22,4-22,9)$ \\
\hline Facteurs de comorbidité c & $47,0(45,8-48,2)$ & $20,4(20,0-20,8)$ & $61,6(61,1-62,1)$ & $29,1(28,9-29,3)$ \\
\hline
\end{tabular}

Abréviations : IMC, indice de masse corporelle; IC, intervalle de confiance

${ }^{a}$ Les trois territoires du Nord canadien : Nunavut, Territoires du Nord-Ouest et Yukon

${ }^{\mathrm{b}}$ Les dix provinces canadiennes : Colombie-Britannique, Alberta, Saskatchewan, Manitoba, Ontario, Québec, Nouveau-Brunswick, Terre-Neuve-et-Labrador, Nouvelle-Écosse et île-du-Prince-Édouard.

Les facteurs de comorbidité : diabète, hypertension artérielle, cardiopathie, asthme, bronchite chronique, emphysème, cancer ou accident vasculaire cérébral

FIGURE 1

Utilisation des services de santé par des Autochtones âgés de 15 ans et plus dans le Norda et dans le Sud ${ }^{\mathrm{b}}$ du Canada par type de fournisseur et en fonction de la présence ou non d'arthrite

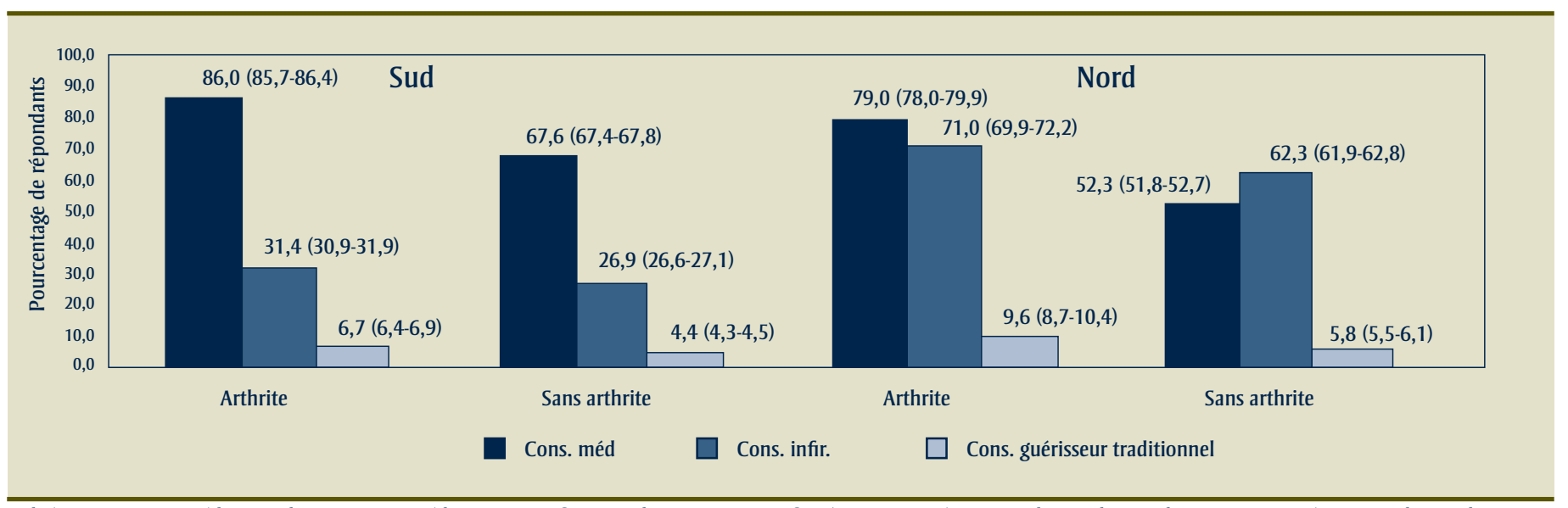

Abréviations : Cons. méd., Consultation avec un médecin; Cons. infir., Consultation avec une infirmière ; Cons. guérisseur traditionnel, Consultation avec un guérisseur traditionnel ${ }^{a}$ Les trois territoires du Nord canadien : Nunavut, Territoires du Nord-Ouest et Yukon

${ }^{\mathrm{b}}$ Les dix provinces canadiennes : Colombie-Britannique, Alberta, Saskatchewan, Manitoba, Ontario, Québec, Nouveau-Brunswick, Terre-Neuve-et-Labrador, Nouvelle-Écosse et Île-du-Prince-Édouard. 
autochtones. Comme on pouvait s'y attendre, la prévalence augmente en fonction de l'âge (tableau 2).

\section{Risques pour la santé}

Le tableau 3 permet de comparer la proportion de fumeurs quotidiens, de personnes obèses et de personnes présentant des facteurs de comorbidité parmi les répondants atteints d'arthrite et ceux qui n'en sont pas atteints.

La prévalence du tabagisme est plus élevée chez les Autochtones du Nord que chez ceux du Sud. Dans le Sud, une association entre le tabagisme quotidien et l'arthrite est observée (rapport de cotes ajusté selon l'âge et le sexe $[R C]=1,58$; IC à $95 \%$ : 1,53 à 1,62), alors que le tabagisme quotidien ne constitue pas un facteur significatif dans le Nord $(\mathrm{RC}=1,05$; IC à $95 \%$ : 0,98 à 1,12). La prévalence de l'obésité (IMC $\geq 30$ ) est supérieure chez les personnes atteintes d'arthrite, et l'association entre l'obésité et l'arthrite est plus étroite chez les Autochtones du Sud $(\mathrm{RC}=1,59$; IC à $95 \%: 1,54$ à 1,64$)$ que parmi ceux du Nord $(\mathrm{RC}=1,36$; IC à $95 \%: 1,26$ à 1,47$)$.

Dans le Nord comme dans le Sud, la proportion de personnes ayant déclaré souffrir d'au moins une autre maladie chronique - diabète, cardiopathie, hypertension artérielle, accident vasculaire cérébral, asthme, bronchite chronique, emphysème ou cancer - est plus élevée chez les personnes atteintes d'arthrite que chez celles qui ne le sont pas (dans le Nord, $\mathrm{RC}=1,88$ avec IC à $95 \%: 1,77$ à 2,00; dans le Sud, RC = 2,55 avec IC à $95 \%: 2,48$ à 2,61).

\section{Utilisation des services de santé}

La proportion de personnes ayant déclaré avoir consulté un professionnel de la santé (infirmière ou médecin de première ligne) ou un guérisseur traditionnel* au cours des douze mois précédant la tenue de l'enquête était plus élevée chez les personnes atteintes d'arthrite que chez celles ne l'étant pas (dans le Nord, $\mathrm{RC}=2,32$ avec IC à $95 \%: 2,10$ à 2,56; dans le Sud, RC $=2,25$ avec IC à $95 \%: 2,17$ à 2,33). Les arthritiques du Nord ont consulté des infirmières et des guérisseurs traditionnels plus souvent que ceux du Sud, et des médecins moins souvent (figure 1).

\section{Conditions sociales}

La proportion de personnes ayant déclaré avoir eu un emploi la semaine avant la tenue de l'enquête (travail rémunéré ou à leur compte) était plus faible parmi les arthritiques que chez les autres. L'association était plus étroite dans le Sud $(\mathrm{RC}=0,58$; IC à $95 \%: 0,57$ à 0,59$)$ que dans le Nord (RC $=0,75$; IC à $95 \%: 0,70$ à 0,79).

\section{Analyse}

La faible prévalence estimée chez les Autochtones du Nord par rapport à ceux du Sud dans le cadre de l'EPA de 2006 correspond aux résultats découlant d'autres enquêtes $^{6,7}$. Par exemple, les analyses de l'ESCC $20002001^{6}$ par Tjekepma ont révélé une prévalence de $10 \%$ chez les Autochtones du Nord, alors que dans le Sud, la prévalence a été établie à $19 \%$ pour les résidents ruraux et à $20 \%$ pour les résidents en milieu urbain. À partir des données de l'ESCC 2005-2006, Lix et coll. ont estimé la prévalence à $12 \%$ dans le Nord et à $20 \%$ dans le Sud ${ }^{7}$. Ces deux études ont également révélé que la prévalence chez les Autochtones est plus élevée que chez les non-Autochtones dans le Sud, mais non dans le Nord. Il convient de souligner que l'ESCC et l'EPA visent les mêmes groupes autochtones - les Métis, les Inuits et les membres des Premières nations vivant hors réserve. Les faibles taux de détection de l'arthrite observés dans le Nord pourraient être attribuables à un accès réduit aux soins de spécialistes; toutefois, la prévalence de l'arthrite est fondée sur l'auto-déclaration, et non sur des diagnostics confirmés en clinique par des rhumatologues. De plus, comme l'arthrite est une maladie chronique, il est fort possible que la maladie ait été diagnostiquée il y a un bon moment, même si l'accès à des soins spécialisés est limité.
Dans des enquêtes telles que l'EPA, l'ESCC et l'ERS, les auto-déclarations dans la catégorie " arthrite et rhumatisme » manquent de précision sur le plan clinique. Les autodéclarations sont aussi limitées par l'incapacité de distinguer les différents types d'arthrites (polyarthrite rhumatoïde, arthrose, etc.). Ce type de mesures brutes est néanmoins utile pour évaluer la santé de la population et les besoins en matière de soins, et surtout pour décrire les tendances dans divers sous-groupes.

La faible prévalence de l'arthrite chez les Autochtones du Nord peut aussi être attribuable à la grande proportion d'Inuits dans la population. (Selon le recensement de 2006, environ $54 \%$ des résidents autochtones des territoires du Nord étaient d'ascendance inuite, alors que seulement $4 \%$ des Autochtones de l'ensemble du Canada le sont ${ }^{14}$.) L'EPA $2001^{9}$ et l'ESCC $20002001^{6}$ ont révélé qu'à l'échelle nationale, la prévalence de l'arthrite est plus faible chez les Inuits que chez les autres Autochtones. Dans le cadre de cette étude, il a été établi que la prévalence de l'arthrite parmi les groupes autochtones (Inuits, Premières nations et autres) vivant dans le Nord est également plus faible que chez ceux vivant dans le Sud (tableau 2).

La raison pour laquelle la prévalence de l'arthrite est plus faible chez les Inuits canadiens que chez les membres des Premières nations n'a pas été clairement établie. La rubrique sous laquelle les participants déclarent souffrir d'arthrite correspond à différents états cliniques dont l'étiologie est disparate. Une analyse des données sur les populations autochtones nord-américaines a révélé que les Inuits ont des taux de spondylarthropathies élevés, tandis que les Américains autochtones ont des taux de polyarthrite rhumatoïde élevés ${ }^{1}$. D’après une étude fondée sur l'examen de dossiers médicaux, le taux de polyarthrite rhumatoïde chez les Inupiat du versant nord de l'Alaska (qui sont liés aux Inuvialuit des Territoires du NordOuest sur le plan culturel et linguistique) est plus élevé que dans certaines tribus

\footnotetext{
*D'après l'EAPA de 2006, un guérisseur traditionnel est défini comme un conseiller, une personne fournissant la médecine traditionnelle, comme des herbes, ou un chef traditionnel ou spirituel reconnu par la communauté.
} 
autochtones américaines, et beaucoup plus élevé que chez les Yupik de la partie ouest de l'Alaska ${ }^{15}$. Selon une étude réalisée récemment en Alaska pour estimer la prévalence de l'arthrite indifférenciée sur le plan clinique et déclarée par les intéressés, la prévalence est plus élevée chez les Autochtones de l'Alaska que dans la population des États-Unis en général; cependant, l'échantillon d'Alaskiens était composé d'une combinaison de Yupik et de tribus autochtones américaines de la partie sud-est de l'État ${ }^{16}$.

Les Autochtones souffrant d'arthrite ont des profils sanitaires défavorables; ils sont davantage susceptibles de fumer quotidiennement, d'être obèses et de souffrir d'autres maladies chroniques. La prépondérance de ces caractéristiques est toutefois différente dans le Nord et dans le Sud, ce qui est révélateur de la prévalence de fond de ces caractéristiques et troubles connexes. L'arthrite peut limiter les possibilités d'emploi, bien que la présente étude ne fournisse aucune indication selon laquelle le faible taux d'emploi serait une conséquence directe de la maladie.

Comme on pouvait s'y attendre, les Autochtones atteints d'arthrite ont davantage tendance à faire appel au système de santé : la proportion d'Autochtones ayant déclaré avoir consulté un médecin, une infirmière ou un guérisseur traditionnel est plus élevée que dans la population en général. Le profil d'utilisation des services cadre avec les différents systèmes en place dans le Nord et le Sud. Il est toutefois impossible de déterminer si l'arthrite est directement en cause dans la plus grande utilisation de services, mais il s'agit d'une explication plausible, compte tenu de la nature de la maladie, de la présence d'autres facteurs de risque, comme le tabagisme et l'obésité ainsi que des facteurs de comorbidité. Dans le Nord, les soins primaires sont principalement fournis par des infirmières dans les centres sanitaires des communautés, et l'accès à des médecins itinérants n'est possible que de manière périodique. Pour beaucoup de patients, consulter un médecin spécialiste comme un rhumatologue implique de voyager par avion.
D’autres travaux sont nécessaires afin de cerner les disparités des fardeaux de l'arthrite chez les populations autochtones du Nord et du Sud. Il faudrait également préciser le diagnostic (p. ex. polyarthrite rhumatoïde, arthrose ou autres troubles musculosquelettiques) et réaliser des analyses distinctes pour les échantillons d'Inuits et ceux des membres des Premières nations dont la population est suffisamment nombreuse dans le Nord. Les conclusions concernant l'arthrite et d'autres maladies chroniques chez Autochtones, tout comme la reconnaissance des différences entre le Sud et le Nord, seront profitables pour la planification de programmes et l'établissement de nouvelles priorités en matière de promotion de la santé. La production de données de qualité et leur transmission aux intervenants concernés, en vue de favoriser l'application des conclusions d'études, contribueront à réduire les disparités sur le plan de la santé.

\section{Remerciements}

La présente étude a été financée par l'Initiative nationale de recherche sur l'arthrite chez les Autochtones du Réseau canadien de l'arthrite (code de projet : 07-NAARI-04).

\section{Références}

1. Peschken CA, Esdaile JM. Rheumatic diseases in North America's indigenous peoples. Semin Arthritis Rheum. 1999;28:368-91.

2. Barnabe C, Elias B, Bartlett J, Roos L, Peschken C. Arthritis in Aboriginal Manitobans: evidence for a high burden of disease. J Rheumatol. 2008;35:1145-50.

3. Ferucci ED, Templin DW, Lanier AP. Rheumatoid arthritis in American Indians and Alaska Natives: a review of the literature. Semin Arthritis Rheum. 2005;34:662-7.

4. Young TK, O’Neil JD, Elias B, Leader A, Reading J, McDonald G. Chapter 3: Chronic diseases. In: First Nations and Inuit Regional Health Survey Final Report [Internet]. MacMillan $\mathrm{H}$, Walsh C, Jamieson E, Crawford A, Boyle M,
Editors. Ottawa (Ont.): First Nations and Regional Health Survey National Steering Committee, 1999 [consulté le 5 juin 2010]. p. 57-81. PDF (11 Mo) téléchargeable à partir du lien : http://rhs-ers.ca/english/pdf/ rhs1997/rhs_1997_final_report.pdf

5. Assembly of First Nations/First Nations Information Governance Committee. First Nations Regional Longitudinal Health Survey (RHS) 2002/2003: results for adult, youth and children living in First Nations communities. $2^{\text {e }}$ éd. mars 2007 [consulté le 5 juillet 2010]. PDF téléchargeable à partir du lien : http://rhs-ers.ca/ english/pdf/rhs2002-03reports/rhs2002-03technicalreport-afn.pdf

6. Tjepkema M. La santé des Autochtones vivant hors réserve. Rapports sur la santé 2002; 13(suppl.): 1-18. [La santé de la population canadienne. Rapport annuel, Ottawa (Ont.): Statistique Canada; $n^{\circ} 82-003$ au catalogue].

7. Lix LM, Bruce S, Sarkar J, Young TK. Facteurs de risque et problèmes de santé chroniques chez les Autochotones et les non-Autochtones. Rapports sur la santé 2009;20: 1-11.

8. Statistique Canada. Langue, tradition, santé, habitudes de vie et préoccupations sociales : Enquête auprès des peuples autochtones de 1991. Ottawa (Ont.): Statistique Canada; 1993 [n 89-533 au catalogue].

9. O’Donnell V, Tait H. Enquête auprès des peuples autochtones de 2001 : premiers résultats - Bien-être de la population autochtone vivant hors réserve. Ottawa (Ont.): Statistique Canada; 2003 [n ${ }^{\circ}$ 89-589-XIE au catalogue].

10. Statistique Canada. L'enquête auprès des peuples autochtones de 2006 : guide des concepts et méthodes. Ottawa (Ont.): Statistique Canada; 2009 [n $\mathrm{n}^{\circ}$ 89-637-X $\mathrm{n}^{\circ} 003$ au catalogue].

11. Oliveria SA, Felson DT, Cirillo PA, Reed JI, Walker AM. Body weight, body mass index, and incident symptomatic osteoarthritis of the hand, hip, and knee. Epidemiology. 1999;10:161-6. 
12. Heliovaara M, Aho K, Aromaa A, Knekt P, Reunanen A. Smoking and risk of rheumatoid arthritis. J Rheumatol. 1993;20:1830-5.

13. Lacaille D, Hogg RS. The effect of arthritis on working life expectancy. J Rheumatol. 2001;28:2315-9.

14. Statistique Canada. Ascendance autochtone (10), région de résidence (6), groupes d'âge (12) et sexe (3) pour la population, pour le Canada, les provinces et les territoires, recensement de 2006 Données-échantillon (20\%). Ottawa (Ont.): Statistique Canada; n 97-558-XCB2006012 au catalogue.

15. Boyer GS, Benevolenskaya LI, Templin DW, Erdesz S, Bowler A, Alexeeva LI, Goring WP, Krylor MY, Mylov NM. Prevalence of rheumatoid arthritis in circumpolar native populations. J Rheumatol. 1998;25:23-9.

16. Ferucci ED, Schumacher MC, Lanier AP, Murtagh MA, Edwards S, Helzer LJ, TomOrme L, Slattery ML. Arthritis prevalence and associations in American Indian and Alaska Native people. Arthritis Rheum. 2008;59:1128-36. 